\title{
Pterodactyl: Trade Study for an Integrated Control System Design of a Mechanically Deployed Entry Vehicle
}

\author{
Antonella I. Alunni ${ }^{*}$, Sarah N. D’Souza ${ }^{\dagger}$, Bryan C. Yount ${ }^{\star}$, Wendy A. Okolo ${ }^{\S}$, Ben E. Nikaido**, Benjamin \\ W.L. Margolis ${ }^{\dagger \dagger}$, and Alan M. Cassell* \\ NASA Ames Research Center, Moffett Field, CA 94035 \\ Breanna J. Johnson ${ }^{\S}$ \\ NASA Johnson Space Center, Houston, TX 77058 \\ Kenneth E. Hibbard $^{* * *}$, Jeffrey D. Barton ${ }^{\dagger \dagger}$, and Gabriel Lopez ${ }^{+*}$ \\ John Hopkins University Applied Physics Laboratory, Laurel, MD 20723 \\ Zane Hays ${ }^{\S \S}$ \\ AMA, Inc., Moffett Field, CA 94035
}

\begin{abstract}
This paper presents a trade study method used to evaluate and down-select from a set of guidance and control (G\&C) system designs for a mechanically deployable entry vehicle (DEV). The Pterodactyl project, funded by NASA's Space Technology Mission Directorate (STMD), was prompted by the challenge to develop an effective G\&C system for a vehicle without a backshell, which is the case for DEVs. For the DEV, the project assumed a specific aeroshell geometry pertaining to an Adaptable, Deployable, Entry Placement Technology (ADEPT) vehicle, which was successfully developed by STMD prior to this study. The Pterodactyl project designed three different G\&C systems for the vehicle's precise entry, which this paper briefly discusses. This paper details the Figures of Merit (FOMs) and metrics used during the course of the project's G\&C system assessment. Each G\&C configuration was traded against the three FOMs categories: G\&C system performance, affordability and life cycle costs, and safety and mission success. The relative importance of the FOMs was determined from the Analytical Hierarchy Process (AHP), which was used to develop weights that were combined with quantitative design metrics and engineering judgement to rank the G\&C systems against one another. This systematic method takes into consideration the project's input while simultaneously reducing unintentional judgement bias and ultimately was used to select a single G\&C design for the project to continue pursuing in the next prototyping and testing phase.
\end{abstract}

\footnotetext{
* Aerospace Engineer, Entry Systems and Vehicle Development Branch, NASA ARC/TSS

$\dagger$ Principal Investigator, Systems Analysis Office, NASA ARC/AA

† Experimental Facility Developer, Engineering Systems Division, NASA ARC/RE

$\S$ Aerospace Research Engineer, Intelligent Systems Division, NASA ARC/TI

** Aerospace Flight Systems Engineer, Systems Analysis Office, NASA ARC/AA

† Graduate Pathways Student, Systems Analysis Office, NASA ARC/AA

* Aerospace Engineer, Entry Systems and Vehicle Development Branch, NASA ARC/TSS

$\S \S$ Aerospace Engineer, Flight Mechanics and Trajectory Design Branch, NASA JSC/EG5

*** Principal Systems Engineer, Space Exploration Sector, JHU/APL

$\dagger \dagger$ Guidance \& Control Engineer, Force Projection Sector, JHU/APL

$\$$ Systems Engineer, Space Exploration Sector, JHU/APL

$\S \S \S$ Research Assistant, Analytical Mechanics Associates, Inc.
} 